\title{
Detection of Acute Myocardial Ischemia by Vessel-Specific Leads Derived from the 12-Lead Electrocardiogram
}

\author{
JY Wang ${ }^{1}$, M Mirmoghisi ${ }^{2}$, JW Warren ${ }^{2}$, GS Wagner ${ }^{3}$, BM Horáček $^{2}$ \\ ${ }^{1}$ Philips Medical Systems, Andover, MA, USA; ${ }^{2}$ Dalhousie University, Halifax, NS, Canada, \\ ${ }^{3}$ Duke University Medical Center, Durham, NC, USA
}

\begin{abstract}
Our aim was to enhance diagnostic performance of ACC/ESC STEMI criteria based on 12-lead ECG by using criteria from 3 derived vessel-specific leads (VSLs). Study data consisted of 12-lead ECGs acquired during angioplasty-induced ischemic episodes due to balloon inflation in, respectively, $\operatorname{LAD}(n=35), R C A(n=47)$, and LCX $(n=17)$ coronary arteries. Three VSLs were derived from 12-lead ECG by previously developed lead transformations. We found that the detection of ischemic state, in any vessel, by means of the ACC/ESC criteria achieved the SE/SP of 60/96\% in the total population, whereas in subgroups sorted by vessel SE/SP were 74/97\% for LAD, 60/94\% for RCA, and 36/100\% for $L C x$. With derived VSLs at $110 \mu \mathrm{V}$ threshold we achieved in total population SE/SP $84 / 93 \%$ and in subgroups $97 / 92 \%$ for LAD, 77/92\% for RCA and 77/100\% for LCx. Thus it appears that VSLs can markedly improve detection of ischemia, especially that caused by LCx artery occlusion.
\end{abstract}

\section{Introduction}

In patients presenting with symptoms of acute coronary syndromes, currently used clinical diagnostic criteria [1-3], based on ST-segment elevation in the 12lead ECG, identify patients suffering from ST-elevation acute myocardial infarction (STEMI) with high specificity, but low sensitivity. Consequently, many false-negative patients do not receive appropriate therapy. We hypothesized that the sensitivity of acute MI detection can be increased by using criteria based on 3 derived vessel-specific leads (VSLs) developed previously from the Dalhousie database of ECGs obtained during controlled acute ischemia [4]. Thus the aim of the present study was to use an independent STAFF3 database from Duke University [5], also consisting of ECGs recorded during controlled acute ischemia, to evaluate the diagnostic capabilities of VSLs and compare them with those that can be achieved by applying STEMI criteria currently used in clinical practice [3].

\section{Methods}

\subsection{Patient population}

The STAFF3 database consists of ECG data for 108 patients originally studied at the Charleston Area Medical Center in West Virginia, who underwent elective ballooninflation percutaneous transluminal coronary angioplasty (PTCA) in one of their main coronary arteries [5]. All of these patients had continuous 12-lead ECG recorded before and throughout the PTCA procedure. Upon initial inspection of plotted ECG tracings, we rejected data of 11 patients with ECGs of substandard quality (i.e., containing artifacts that could not be eliminated or reduced by the usual ECG processing techniques). Therefore, we were left with test-set population consisting of 97 patients. The balloon-inflation periods for these patients ranged from 1'30" to 7'17", with a mean of 4'37"; these occlusion periods were considerably longer than those used in Dalhousie PTCA protocol [4]. For each patient balloon-inflation PTCA recording (denoted PR) began just before the balloon inflation and continued for the entire occlusion-reperfusion period.

\subsection{ECG acquisition and processing}

The standard 12-lead ECG was recorded digitally for each participant of the study by the Siemens-Elema AB (Solna, Sweden) ECG cart at 1,000 Hz sampling rate with an amplitude resolution of $0.6 \mu \mathrm{V}$ for the least-significant bit [5]. For each patient we selected only one PR recording for analysis (if there indeed were multiple inflations in the same artery for that patient), except in 2 patients with double-artery treatment for whom we used one PR recording for each occluded artery; thus the total number of ischemic episodes documented by 12-lead ECG was 99; 35 of these episodes were due to left anterior descending (LAD) coronary artery occlusion, 47 due to right coronary artery (RCA) occlusion, and 17 due to left circumflex (LCx) coronary artery occlusion. Subsequent ECG processing was done at Dalhousie University on an RS/6000 computer (IBM Corp, Armonk, 
NY). For an entire duration of each PR recording we calculated 3 VSLs from 8 independent leads of 12-lead ECG [4], and we identified one 10-second interval as a "baseline state" and another 10-second interval (just before reperfusion) as an "ischemic state." After signal smoothing (a running median filter of 3 points) on each lead, QRS onset was determined for each beat, to establish the local baseline, and the local QRS offset was designated as $\mathrm{J}$ point. Local baseline was defined as mean amplitude of 10 samples centered at $10 \mathrm{~ms}$ before the local QRS onset. To measure ST deviation ( $\uparrow S T$ ) of each beat, the difference between amplitude at J point and that at the local baseline was taken. The ST deviations over the entire time course of each ischemic episode were then plotted as histograms for 15 leads. Figs. 1-3 show examples of these plots for ischemic episodes caused by the occlusion of RCA, LAD, and LCx coronary arteries, respectively.

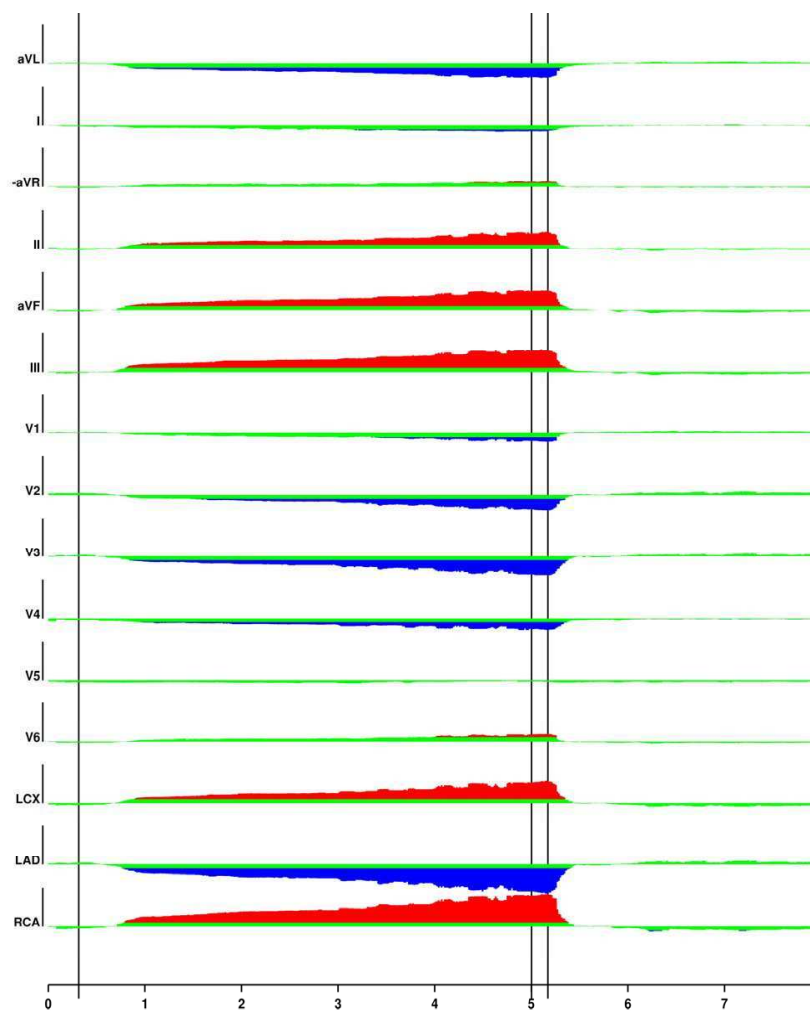

Figure 1. Beat-by-beat histograms of ST deviation in 15 ECG leads for a patient with RCA occluded. Rows correspond to 12 standard leads in Cabrera sequence [6] followed by 3 VSLs (LCX, LAD, RCA); time axis is calibrated in minutes; vertical lines define "baseline state" and "ischemic state." Red bars indicate $\uparrow \mathrm{ST} \geq 100 \mu \mathrm{V}$, blue bars $\downarrow \mathrm{ST} \leq-100 \mu \mathrm{V}$, and green bars $\uparrow \mathrm{ST}$ within 100 $\mu \mathrm{V}$ bounds.

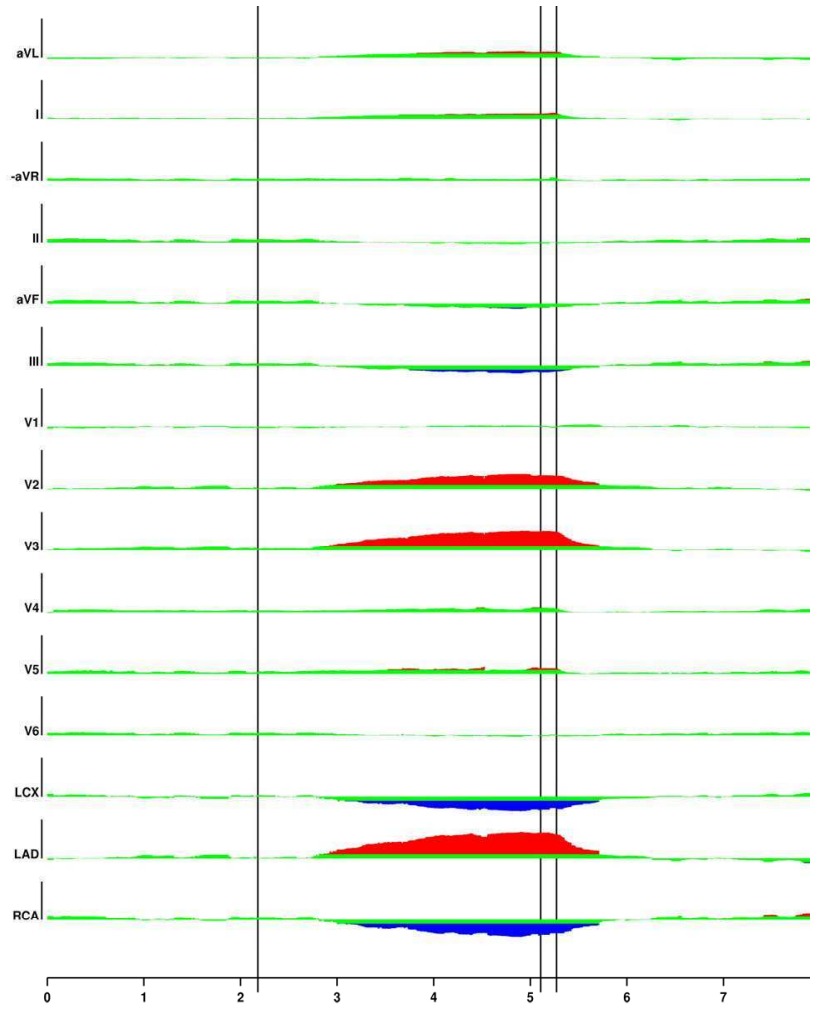

Figure 2. Beat-by-beat histograms of ST deviation in 15 ECG leads for a patient with LAD coronary artery occluded. Same layout as in Fig. 1.

\subsection{ACC/ESC criteria for STEMI}

We implemented ACC/ESC STEMI criteria as follows: If the amplitude at the $\mathrm{J}$ point in both leads of the contiguous pairs (V1, V2), (V2, V3) was $\geq 200 \mu \mathrm{V}$ or in the pair (V3, V4) in lead $\mathrm{V} 3 \geq 200 \mu \mathrm{V}$ and in lead V4 $\geq$ $100 \mu \mathrm{V}$ or in any of the contiguous lead pairs (V4, V5), (V5, V6), (aVL, I), (I, -aVR), (-aVR, II), (II, aVF), (aVF, III), (III, $-\mathrm{aVL}$ ) both J-point amplitudes were $\geq 100 \mu \mathrm{V}$, then the STEMI criteria were met. Otherwise the STEMI criteria were not met. The leads $-\mathrm{aVR}$ and $-\mathrm{aVL}$ denote the negated leads aVR and aVL, respectively.

\subsection{Assessment of diagnostic performance}

The ability of diagnostic criteria to detect acute ischemia was assessed by diagnostic classification that separated the "ischemic" from the "non-ischemic" state using, in turn, the ACC/ESC and VSL criteria (for the latter threshold was incremented in $1-\mu \mathrm{V}$ steps within the range of $0-500 \mu \mathrm{V})$. Using a bootstrap method with replacement, we generated SE and SP values for 1,000 bootstrap trials [7] and the mean value $\pm 95 \%$ confidence limits were plotted and compared (Figs. 4-7). 


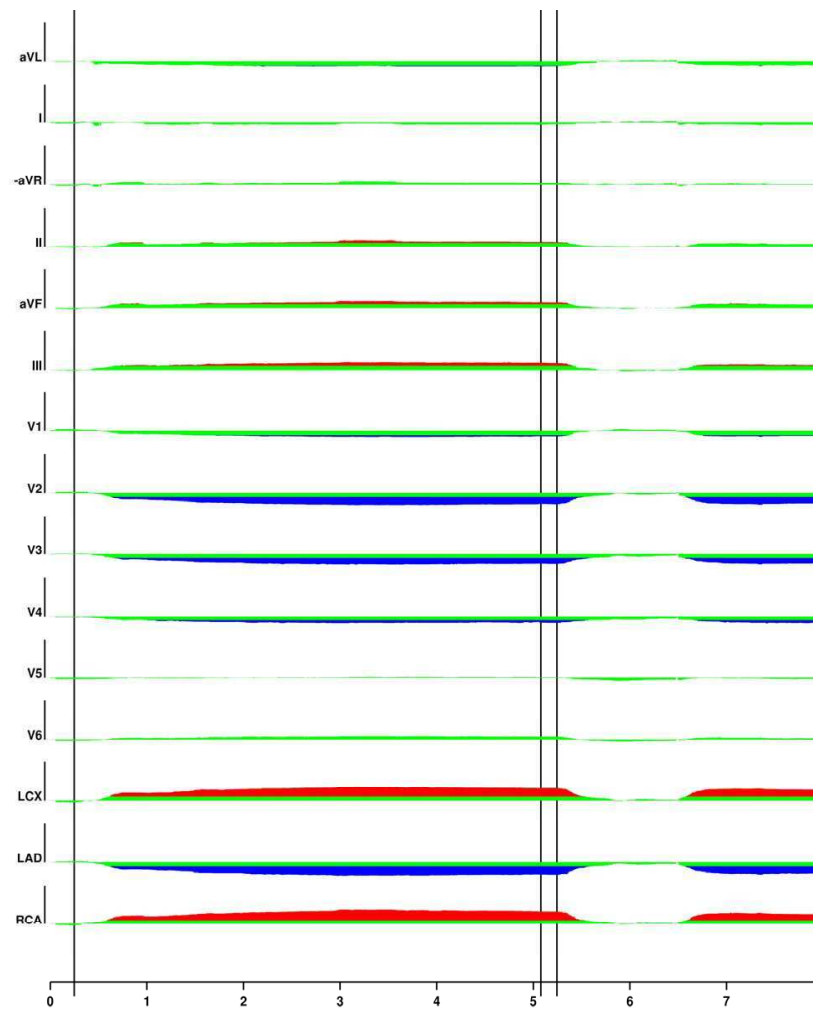

Figure 3. Beat-by-beat histograms of ST-deviation in 15 ECG leads for a patient with LCx coronary artery occluded. Responses to the first and the beginning of the second balloon inflation are shown. Same layout as in Fig. 1.

\section{Results}

A total patient population $(n=97)$ included "responders" ( $n=83)$ who responded to balloon inflation by $\uparrow \mathrm{ST} \geq 100 \mu \mathrm{V}$ in one or more leads of the 12-lead ECG, and "nonresponders" $(n=14)$ with no $\uparrow S T \geq 100$ $\mu \mathrm{V}$ in the 12-lead ECG. We found that in a total patient population the detection of ischemic state, in any vessel, by means of the ACC/ESC criteria achieved the SE/SP of $60 / 96 \%$ (Fig. 4); whereas in subgroups sorted by vessel SE/SP were $74 / 97 \%$ for LAD (Fig. 5), 60/94\% for RCA (Fig. 6), and 36/100\% for LCx (Fig. 7). With derived VSLs at $110 \mu \mathrm{V}$ threshold we achieved in total population SE/SP 84/93\% (Fig. 4) and in subgroups 97/92\% for LAD (Fig. 5), 77/92\% for RCA (Fig. 6) and 77/100\% for LCx (Fig. 7). In addition to detecting acute ischemia due to occlusion of any vessel, VSLs were capable of identifying, at $110 \mu \mathrm{V}$ threshold, the culprit vessel causing ischemia with SE/SP of $95 / 92 \%$ for LAD, $63 / 94 \%$ for RCA, and $70 / 100 \%$ for LCx.

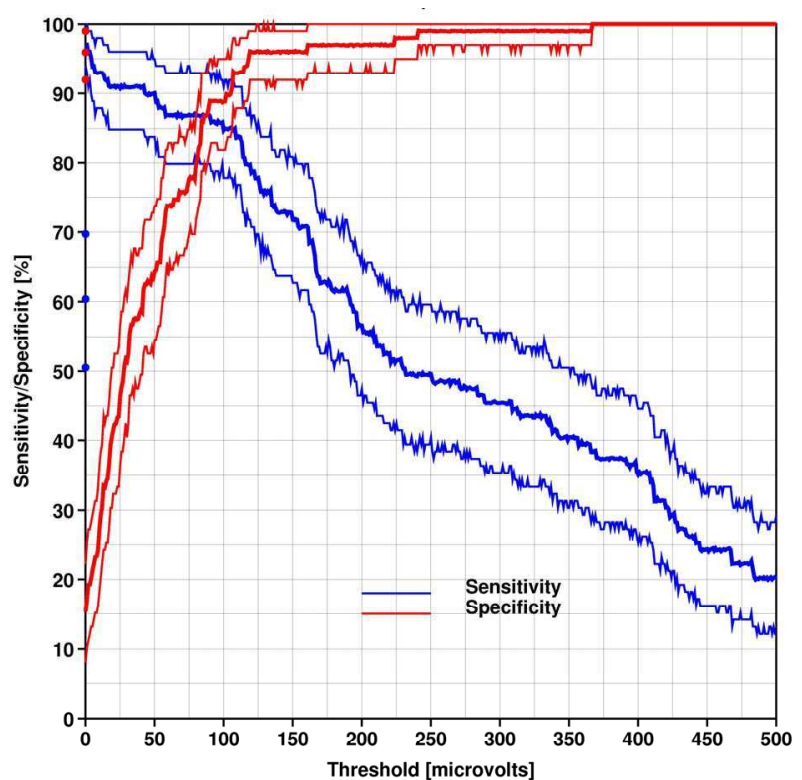

Figure 4. Detection of acute ischemia by ACC/ESC vs VSL criteria: SE and SP of J-point amplitudes for total population $(n=99)$. Mean values and $95 \%$ confidence limits obtained by bootstrap method are plotted for VSLs as a function of threshold and for ACC/ESC STEMI as solid circles on $y$ axis $(\mathrm{SE}=60 \%$; $\mathrm{SP}=96 \%)$. Note that for VSLs at $110-\mu \mathrm{V}$ threshold $\mathrm{SE}=84 \%$ and $\mathrm{SP}=93 \%$; for $175-\mu \mathrm{V}$ threshold $\mathrm{SE}=63 \%$ and $\mathrm{SP}=97 \%$.

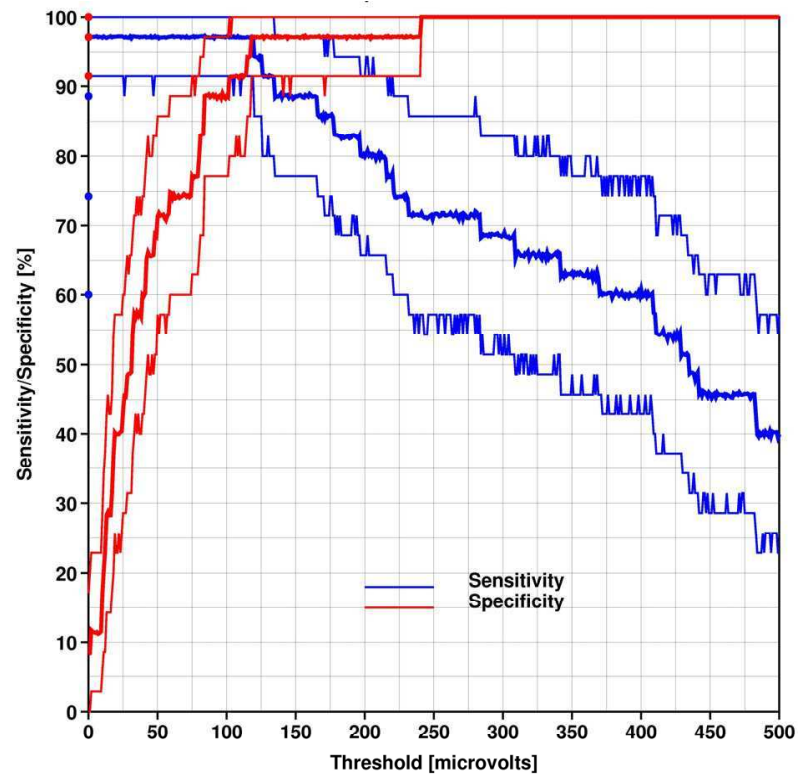

Figure 5. Detection of acute ischemia by ACC/ESC vs VSL criteria: SE and SP of J-point amplitudes for LAD subgroup $(n=35)$. Same layout as in Fig. 4. 


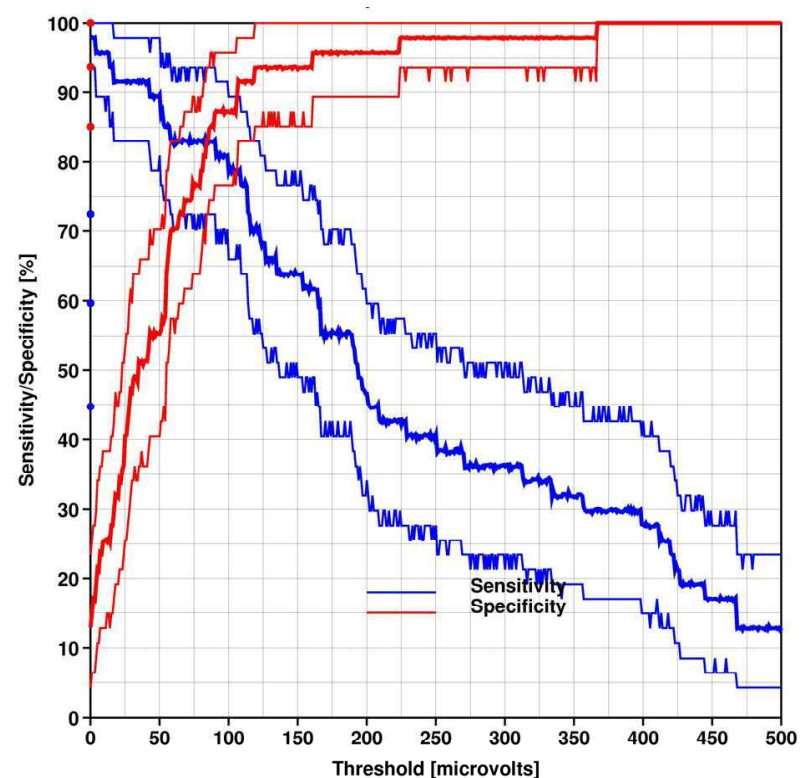

Figure 6. Detection of acute ischemia by ACC/ESC vs VSL criteria: SE and SP of J-point amplitudes for RCA subgroup $(n=47)$. Same layout as in Fig. 4.

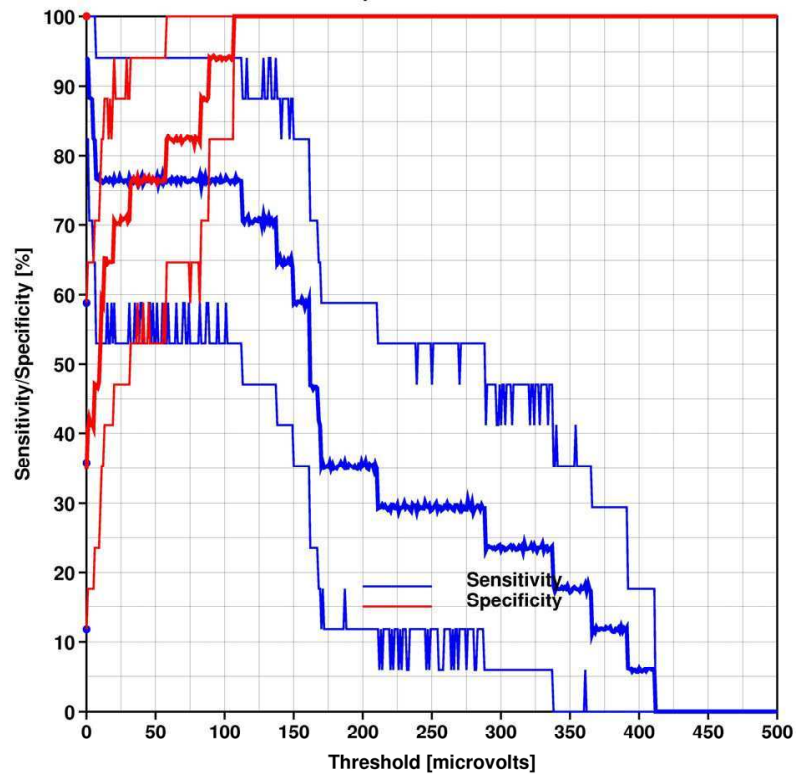

Figure 7. Detection of acute ischemia by ACC/ESC vs VSL criteria: SE and SP of J-point amplitudes for LCx subgroup $(n=17)$. Same layout as in Fig. 4.

\section{Discussion}

Our results, which will need validation on larger patient population, suggest that VSLs derived from the standard 12-lead ECG can identify acute myocardial ischemia in any vessel with better diagnostic performance ( $\mathrm{DP}=\mathrm{SE} / 2$
$+\mathrm{SP} / 2$ ) than existing ACC/ESC criteria applied to the 12 standard leads. This DP increase was $10 \%$ for the total population and $9 \%, 7 \%$, and $20 \%$ for $\mathrm{LAD}, \mathrm{RCA}$, and LCx subgroups, respectively. In addition, VSLs offer a simple identification of the coronary artery responsible for ischemia. In particular, vessel-specific leads can very significantly improve detection of acute ischemia caused by the occlusion of the LCx artery, which is frequently missed by conventional 12-lead ECG criteria [8].

\section{Acknowledgements}

Support for studies at Dalhousie University was provided by the Heart \& Stroke Foundation of Nova Scotia and by the Canadian Institutes of Health Research.

\section{References}

[1] Antman EM, et al. ACC/AHA guidelines for the management of patients with ST-elevation myocardial infarction-executive summary. J Am Coll Cardiol. 2004;44:671-719.

[2] The Joint European Society of Cardiology/American College of Cardiology Committee: Myocardial infarction redefined-A consensus document. Eur Heart J. 2000; 21:1502.

[3] Giugliano RP and Braunwald E. American College of Cardiology; American Heart Association. 2004 ACC/AHA guidelines for the management of patients with STEMI: the implications for clinicians. Nat Clin Pract Cardiovasc Med. 2005;2:114-5.

[4] Horáček BM, Warren JW, Penney CJ, et al. Optimal electrocardiographic leads for detecting acute myocardial ischemia. J Electrocardiol. 2001;34(Suppl):97-111.

[5] Garcia J, Wagner G, Sörnmo L, Lander P, Laguna P. Identification of the occluded artery in patients with myocardial ischemia induced by prolonged percutaneous transluminal coronary angioplasty using traditional vs transformed ECG-based indexes. Comput Biomed Res. 1999;32:470-82.

[6] Cabrera E. Bases électrophysiologiques de l'électrocardiographie, des applications cliniques. Masson, Paris, 1948.

[7] Efron B, Tibshirani RJ. An introduction to the bootstrap. Chapman and Hall, New York, 1993.

[8] Wung SF, Drew BJ. New electrocardiographic criteria for posterior wall acute myocardial ischemia validated by percutaneous transluminal coronary angioplasty model of acute myocardial ischemia. Am J Cardiol. 2001;87:970-4.

Address for correspondence:

John J. Wang

Philips Medical Systems

3000 Minuteman Road, MS-0455

Andover, MA 01810-1099

USA

E-mail: john.j.wang@philips.com 